\title{
Estudo crítico do tratamento de 196 casos de fratura diafisária de rádio e ulna em cães ${ }^{1}$
}

\author{
Cássio Ricardo Auada Ferrigno $0^{2^{*}}$, Alexandre Schmaedecke ${ }^{3}$, Christian \\ Patané ${ }^{4}$, Daniel Castelo Branco Baccarin ${ }^{4}$ e Lucia Maria Gedes Silveira ${ }^{2}$
}

\begin{abstract}
Ferrigno CRA., Schmaedecke A., Pantané C., Baccarin D.C.B. \& Silveira L.M.G. 2008. [A critical study about the treatment of 196 cases of diphiseal radial and ulnar fractures in dogs.] Estudo crítico do tratamento de 196 casos de fratura diafisária de rádio e ulna em cães. Pesquisa Veterinária Brasileira 28(8):371-374. Departamento de Cirurgia, Faculdade de Medicina Veterinária e Zootecnia, Universidade de São Paulo, Av. Prof. Dr. Orlando Marques de Paiva 87, São Paulo, SP 05508-900, Brazil. E-mail: cassioaf@usp.br

The results from three osteosynthesis methods in cases of proximal, mid and distal fractures of radio and ulna in dogs were compared: the skeletal external fixation, plates and screws and the external immobilization. Shaft fractures of radius and ulna in dogs are frequent among the orthopedic morbid cases in Veterinary Medicine and their treatment deserves special attention, due to the high number of unsuccessful cases observed. The demographic data with reference to distribution and classification of fractures, as well as the method used for osteosynthesis and its consequences, were tabulated and the data were statistically compared. As results, from the 196 fractures observed, patients with up to 6.0 kilograms were the most affected (46.4\%) with lesions mainly in the distal third $(64.93 \%$ of the fractures). Animals that have been operated with smaller interval between the fracture occurrence and the surgery showed better results. There was a significant difference between osteosynthesis by external fixators and screwed plates when compared to external immobilization, independent on the fracture site.
\end{abstract}

INDEX TERMS: Fractures, radius and ulna, diaphysis, osteosynthesis, dogs.

RESUMO.- Fraturas diafisárias de rádio e ulna em cães são freqüentes dentro dos quadros mórbidos ortopédicos em Medicina Veterinária, e seu tratamento merece especial atenção, face aos muitos quadros de insucesso observados. Foram comparados neste estudo os resultados de três métodos de osteossíntese, sendo estes a fixação esquelética externa, o uso de placas e parafu-

\footnotetext{
${ }^{1}$ Recebido em 5 de maio de 2008.

Aceito para publicação em 17 de julho de 2008.

2 Departamento de Cirurgia, Faculdade de Medicina Veterinária e Zootecnia (FMVZ), Universidade de São Paulo (USP), Av. Prof. Dr. Orlando Marques de Paiva 87, Cidade Universitária, São Paulo, SP 05508-270, Brasil. *Autor para correspondência: cassioaf@usp.br

${ }^{3}$ Departamento de Medicina Veterinária, Faculdade de Medicina Veterinária, Universidade Federal do Paraná, Rua dos Funcionários 1540, Juvevê, Curitiba, PR 80035-050, Brasil.

${ }^{4}$ Médico Veterinário Autônomo, Rodovia SC 405, 4116 apto 301, Campeche, Florianópolis, SC 88065- 000, Brasil.
}

sos e a imobilização externa, em quadros de fraturas de terço proximal, médio e distal de rádio e ulna em cães. Os dados demográficos e referentes à distribuição e classificação das fraturas, bem como o método utilizado para osteossíntese e suas conseqüências foram tabulados e os dados comparados estatisticamente. Como resultados, das 196 fraturas acompanhadas, pacientes com até $6,0 \mathrm{~kg}$ foram os mais acometidos $(46,4 \%)$ com lesões principalmente no terço distal $(64,93 \%$ das fraturas). Animais que foram operados com intervalo menor entre a ocorrência da fratura e a cirurgia demonstraram meIhores resultados. Houve diferença significativa entre as osteossínteses por fixadores externos e placas parafusadas, quando comparados à imobilização externa, independentemente da região fraturada.

TERMOS DE INDEXAÇÃO: Fraturas, rádio e ulna, diáfise, osteossíntese, cães. 


\section{INTRODUÇÃO}

As fraturas de radio ulna constituem de $8,5-10,7 \%$ de todas as fraturas que acometem os cães e gatos (SummerSmith \& Cawley 1970), sendo considerada a terceira forma de fratura mais comum nos cães. (Brianza et al. 2006). Apenas um ou os dois ossos do antebraço podem estar fraturados em apenas um local ou com fraturas múltiplas combinadas (Sumner-Smith, 1970, Lappin et al. 1983).

O tratamento impreciso das fraturas destes ossos é, por vezes, o fator determinante a não união ou má união, causando desvios e incongruências ósseas, com efeitos maléficos à deambulação do animal (Sumner-Smith \& Cawley 1970, Deangelis et al. 1973, Lappin et al. 1983, Vaughan 1984, Bellah 1987). Este fato pode ser explicado por certas características inerentes a estes ossos, como pouco recobrimento de tecido muscular, canal medular pequeno e pouca vascularização, sendo estas apontadas como causas principais de complicações, sejam após cirurgias ou em tratamentos conservadores (Sumner-Smith \& Cawley 1970, Deangelis et al. 1973, Lappin et al. 1983, Vaughan 1984, Bellah 1987, Denny 1990, Egger 1993, Turner 1995).

O fator racial tem influência nas complicações. Nas raças grandes ou gigantes os vetores de forças, causados pelo peso do animal na fratura e no material de fixação é grande (Lappin et al. 1983, Bellah 1987, Denny 1990, Egger 1993, Turner 1995). Já nas raças pequenas, Vaughan (1984) relata até 60\% de não união óssea, causada geralmente pela pobre vascularização regional. Brianza et al. (2006) citam ainda diferenças morfométricas que justificam a predisposição das raças pequenas em desenvolver soluções de continuidade dos ossos em questão.

O radio é o osso no qual se deposita a maior parte do peso sustentado pelo antebraço. Por suas características anatômicas, indica-se o tratamento de fraturas de sua diáfise com a utilização de placas, por seu córtex cranial quase plano, e fixadores externos, pela facilidade de abordagem nos planos cranial, lateral e medial (Deangelis et al. 1973, Lappin et al. 1983, Bellah 1987, Egger 1993, Turner 1995).

O tratamento conservativo, com imobilização externa como agente de suporte, é recomendado quando o animal não for de raças miniaturas, houver pelo menos $50 \%$ de contato entre as superfícies ósseas e não houver desvios angulares significativos (Lappin et al. 1983, Denny 1990, Egger 1993).

Este estudo retrospectivo visou analisar as características demográficas, tempo de retorno à deambulação normal e qualidade dos resultados do tratamento de fraturas diafisárias de rádio e ulna em cães submetidos à osteossíntese por placa e parafusos, fixadores externos ou imobilização externa.

\section{MATERIAL E MÉTODOS}

Foram catalogados 231 casos de fraturas de rádio e ulna em cães, atendidos em período de três anos. Destes, foram selecionados 196 casos de fraturas diafisárias, cujos pacientes foram acompanhados por todo o período de recuperação pósoperatória. Somente foram utilizados para este estudo os pacientes que apresentaram todas as avaliações pós-operatórias.

Os dados referentes à raça, idade, peso, local, tipo de fratura, complicações, tempo para a união e finalmente a qualidade de utilização do membro na deambulação foram tabulados, sendo analisados estatisticamente. Os casos analisados foram divididos, segundo a disposição da fratura em relação ao rádio, em proximal, médio e distal. Para esta consideração, foi tomado como parâmetro o comprimento total do osso, sendo igualmente subdividido em três partes. Desta divisão, os casos foram subagrupados entre aqueles que apresentaram consolidação óssea adequada, com desvio angular inferior a $10^{\circ}$ e deambulação adequada (BOM), aqueles que apresentaram desvios angulares entre $10^{\circ}$ e $30^{\circ}$ ou má união (DESVIO ANGULAR) e, finalmente os pacientes que não apresentaram sinais radiográficos de consolidação óssea e impotência funcional do membro afetado ou desvios angulares superiores a $30^{\circ}$ (NÃO UNIÃO), conforme utilizado por Rovesti et al. (2007).

Foi considerado como quadro de não união óssea aquele cuja imagem radiográfica não apresentava alteração compatível com os sinais de consolidação por período de 60 dias após o procedimento cirúrgico.

Os dados foram submetidos à análise de variância, sendo comparados os efeitos de tratamento (penso, fixador externo e placa) da idade (0-6 meses, 6 meses a 1 ano, 1-2 anos, 2-5 anos, 5-8 anos ou mais de 8 anos), do peso (0-6 kg, 6-15 kg, $15-25 \mathrm{~kg}$, mais que $25 \mathrm{~kg}$ ) e do local de fratura (distal, médio e proximal) em relação ao tempo de consolidação e complicações. Para comparação entre as médias, foi utilizado o teste de Tukey. Para a comparação dos dados relativos à análise dos grupos, comparando as porcentagens obtidas com as esperadas, foi utilizado o teste de qui-quadrado.

\section{RESULTADOS}

Foram foram analisados por este estudo 196 pacientes, dos quais, 82 cães foram submetidos a tratamento com imobilização externa (penso), 26 com fixadores externos, $88 \mathrm{com}$ placas e parafusos.

Os resultados relacionados aos dados demográficos demonstraram que os pacientes com até $6,0 \mathrm{~kg}$ foram os mais acometidos $(46,4 \%)$ com lesões principalmente no terço distal $(64,93 \%$ das fraturas). Os grupos de cães com mais de $6,0 \mathrm{Kg}$ de não apresentaram diferença de incidência, sendo de 16,3\% dos cães com peso de 6,0-15,0 $\mathrm{kg}, 17,4 \%$ dos cães com peso de 15,0-25,0 kg e 19,9\% de cães com peso acima de 25,0 kg.

As fraturas simples fechadas foram as mais freqüentes, com a porcentagem de $(81,38 \%)$ seguidas das fraturas simples abertas $(10,38 \%)$, das fraturas cominutivas fechadas $(6,49 \%)$ e por ultimo as fraturas cominutivas abertas $(1,73 \%)$. Fraturas em terço proximal perfizeram $5,61 \%$ do total, as de terço médio $28,06 \%$ e as de terço distal, amplamente superiores, corresponderam a $66,33 \%$ do total de casos deste estudo.

Considerando apenas a técnica utilizada, independente do tipo ou posicionamento da fratura, os tratamentos com placas ou fixadores externos mostraram maior sucesso que as imobilizações (pensos), apresentando, respectivamente $78,41 \%, 69,23 \%$ e $46,24 \%$ de bons resultados, 
ou seja, com deambulação normal do paciente e consolidação sem sinais de desvios angulares.

$O$ estudo estatístico das influencias da idade $(p=0,15)$ e do peso $(p=0,156)$, na consolidação não apresentaram diferença significativa.

A análise de variância da relação entre inicio do tratamento após a fratura e tempo de utilização do membro operado (apoio) foi significativo ( $p<0,001)$, mostrando que animais que foram operados com intervalo menor entre a ocorrência da fratura e a cirurgia demonstraram melhores resultados.

Não houve efeito da idade em relação aos resultados após o tratamento $(p=0,154)$ e peso em relação ao inicio da utilização do membro afetado $(p=0,084)$.

Relativos às fraturas de terço proximal de rádio e ulna, a análise de qui-quadrado demonstrou diferença entre os dados obtidos e os esperados para os tratamentos onde se utilizou imobilização esparadrapada, sendo que o grupo de pacientes que apresentaram resultado bom ficou abaixo do valor esperado, se relacionados aos resultados dos grupos dos pacientes submetidos à osteossíntese por fixador externo ou placa.

Para as fraturas de terço médio de rádio e ulna, os pacientes submetidos à imobilização apresentaram valores superiores aos esperados em relação a não união óssea, se comparados aos grupos dos pacientes submetidos à osteossíntese por fixador externo ou placa.

E para os pacientes do grupo de fraturas de terço distal de rádio e ulna, os que foram submetidos à imobilização esparadrapada apresentaram resultados inferiores à expectativa, em relação aos demais grupos, para o conceito bom e resultados superiores à expectativa para os conceitos desvio angular e não união. O grupo de animais submetidos à fixação externa apresentou resultados superiores ao esperado para o conceito não união, e o grupo de pacientes submetido à osteossíntese por placas apresentou número de casos superior em relação à expectativa para o conceito desvio angular.

\section{DISCUSSÃO}

As fraturas de rádio e ulna de animais de companhia são moléstias ortopédicas de grande incidência na rotina clínico-cirúrgica em medicina veterinária. O manejo destas fraturas envolve coaptação externa ou estabilização cirúrgica. Com a difusão de conhecimento e desenvolvimento de novos equipamentos e técnicas, já não existe espaço para os procedimentos utilizados ate pouco tempo atrás, com a utilização de pinos intramedulares como técnica de osteossíntese, sob a alegação de ser procedimento menos oneroso. O que se vê é a baixa capacidade desta técnica no bloqueio das forças atuantes no foco de fratura, não justificando o grau de invasividade da técnica, além de promover lesão inaceitável na articulação carpo-radial para inserção medular da haste, conforme descrito por Harasen (2003).

Referentes aos dados demográficos, os resultados demonstraram que as raças miniaturas foram as mais acometidas $(54,14 \%)$ com lesões principalmente no terço distal $(64,93 \%$ das fraturas de radio ulna em raças miniaturas), estas também apresentaram o maior número de não união óssea (31,7\%). Resultados comprovados anteriormente por Sumner-Smith \& Cawley 1970, Deangelis et al. 1973, Lappin et al. 1983, Vaughan 1984, Bellah 1987, Denny 1990, Egger 1993, Turner 1995 que relatam a característica de dificuldade de imobilização, pouca cobertura de tecidos moles sobre o osso, canal medular estreito, pouca vascularização e demora na cicatrização inerentes a estas raças.

Placas e fixadores externos se tornaram as técnicas cirúrgicas de eleição, enquanto as imobilizações esparadrapadas se restringem a alguns casos específicos (Lappin et al. 1983, Denny 1990, Egger 1993).

Em relação à utilização de imobilizações de coaptação externa, embora seja esta a forma mais antiga e ainda utilizada sem critérios em todas as clínicas de pequenos animais, deve ser evitada em fraturas de rádio e ulna de animais de raças miniaturas (Sumner-Smith 1970, Vaughan 1984, Egger 1993) pois estes apresentam severas complicações, incluindo mau alinhamento ou não união na maioria dos casos em que foram submetidos ao tratamento de fraturas com imobilização externa. Essas complicações, também relatadas por Herron (1974) e Lappin et al. (1982), ocorrem normalmente devido a dificuldades de manter o correto alinhamento do foco de fratura, causado pela tensão do flexor do carpo e do músculo flexor digital deslocam os fragmentos caudalmente e lateralmente. No presente estudo, a utilização de coaptação externa se mostrou menos efetiva em todas as comparações dos pacientes submetidos a esta técnica, em relação àqueles onde se aplicaram as técnicas de fixação externa ou placa, independente da região fraturada.

Estes animais, na maioria das vezes, foram casos encaminhados por outros veterinários que já haviam tratado as fraturas anteriormente com pensos esparadrapados sem sucesso. Resultados que são similares aos de Sumner-Smith (1970), Deangelis et al. (1973), Lappin et al. (1983), Vaughan (1984), Bellah (1987), Egger (1993) e Turner (1995).

Porém, os dados de desta pesquisa mostram que os pensos e talas podem ser empregados em cães de médio porte, jovens em fraturas simples fechadas de terço médio de rádio e ulna, com alguma segurança, devido ao seu metabolismo que permite cicatrização óssea mais rápida e sem problemas de vascularização. Afirmações similares foram relatadas em trabalhos de Lappin et al. (1983) Denny (1990) e Egger (1993).

Em animais muito pesados, os dados deste artigo não preconizam a utilização de pensos e sim a cirurgia para colocação de fixadores externos ou placa, pois houve tendência estatística de feito negativo do peso sobre o apoio $(p=0,084)$, como descrito por Lappin et al. (1983).

Os fixadores externos foram introduzidos pelo Dr. E. Ehmer e a companhia Kirschner 1947 (Bradley \& Rouse 1980). E desde então os fixadores com duas barras e os aparelhos com uma única barra tem sido muito utilizados em fraturas de radio ulna em varias espécies animais in- 
cluindo o cão e gato. Piermattei (1978) cita como sendo os fixadores externos o melhor critério de fixação de fraturas por suas características de facilidade de aplicação, capacidade de manter os fragmentos ósseos por período longos, e a principal característica, injúria vascular mínima.

Bons resultados puderam ser observados com a utilização desta técnica, com a maioria dos pacientes retornando à deambulação normal, sem restrições, e em tempo satisfatório. No entanto, em alguns casos, principalmente em animais pequenos, os fixadores se mostraram menos eficazes que as placas, principalmente em fraturas de terço distal dos ossos em questão, provavelmente pelo maior peso do implante, com conseqüente incapacidade de deambulação adequada por parte dos pacientes e pouco estímulo no foco de fratura. A biomecânica do implante em relação a este tipo de fratura também deve ser levada em consideração, uma vez que a proximidade da transposição dos pinos do fragmento distal pode não promover a adequada estabilização do foco da fratura.

Este método em nosso entendimento é o melhor tipo de tratamento para fraturas expostas, devido à menor injuria causada nos tecidos moles adjacentes ao osso, associado à pequena abordagem e exposição óssea necessária para a sua instituição, além de manter contato mínimo entre implante e osso, evitando colonização de microorganismos na interface osso metal.

Já as osteossínteses por placa e parafusos, muito populares no tratamento de fraturas de rádio e ulna de seres humanos (Anderson et al. 1975) e cães (Sumner-Smith 1970) apresentam resultados satisfatórios, com boa deambulação e rápido retorno à função do membro, conforme demonstrado por Sumner-Smith (1970), Lappin et al. (1983), Vaughan (1984), Egger (1993), principalmente em raças miniaturas.

As raças miniaturas foram as mais acometidas por fraturas de rádio e ulna, resultados semelhantes a relatos de Sumner-Smith (1970), Piermattei (1978) e Lappin et al. (1983), e na maioria dos casos foram utilizadas placas com êxito.

A utilização de placas em casos em animais com peso abaixo que $6 \mathrm{~kg}$, nesta pesquisa mostrou-se como fixação óssea mais eficaz, indicada principalmente pela capacidade de conter as forças de encurvamento, rotação e distração efetivamente, e por ter, comparativamente, peso do implante menor, que os fixadores externos. Resultados estes semelhantes aos de Lappin et al. (1983).

As placas obtiveram resultados estatísticos melhores que os fixadores externos, com um total de $78,41 \%$ de êxito contra $69,23 \%$ dos fixadores externos, provavelmente porque os fixadores foram mais utilizados em fraturas abertas ou com alta cominução. Resultados semelhantes aos relatados por Sumner-Smith (1970), Brinker et al. (1986), Bellah (1987), Denny (1990), Egger (1993) e Turner (1995).

\section{CONCLUSÕES}

O presente trabalho possibilitou concluir que para o tratamento fraturas de rádio e ulna de cães de pequeno porte, assim como fraturas instáveis ou fraturas em que não houve o pronto atendimento, especial cuidado em relação à escolha do método de osteossíntese deve ser tomado. Confirmou-se que a osteossíntese por placa parafusada ou fixação esquelética externa apresentam meIhores resultados, independentemente do padrão ou disposição da fratura, fato este ainda mais relevante em cães de peso inferior a $6,0 \mathrm{~kg}$.

Agradecimentos.- À Fundação de Amparo à Pesquisa do Estado de São Paulo-FAPESP, pelo financiamento do projeto.

\section{REFERÊNCIAS}

Anderson L.D., Tooms R.E. \& Park III W.I. 1975. Compression- plate fixation in acute diaphyseal fractures of the radius and ulna. J. Bone Joint Surg. 57:287-297.

Bellah R.J. 1987. Use of a distal hook plate for treatment of a distal radial fracture in a dog. Vet. Surg. 16(4/8):278-282.

Bradley R.L. \& Rouse G.P.1980. External skeletal fixation using the through-and-through Kirschner Ehmer splint. J. Am. Anim. Hosp. Assoc. 6:523-530.

Brianza S.Z., Delise M., Maddalena Ferraris M., D’amelio P. \& Botti P. 2006. Cross-sectional geometrical properties of distal radius and ulna in large, medium and toy breed dogs. J. Biomech. 39(2):302311.

Brinker W.O., Piermatei M.L. \& Flo G.L. 1999. Fraturas do radio e da ulna, p.301-321. In: __ (ed.), Manual de Ortopedia e Tratamento das Fraturas dos Pequenos Animais. Manole, São Paulo.

Deangelis M.P., Olds R.B. \& Stoll S.G.1973. Repair of fractures of the radius and ulna in small dogs. J. Am. Anim. Hosp. Assoc. 19:436441.

Denny H.R. 1990. Pectoral limb fractures, p.357-387. In: Whittick W.G. (ed.), Canine Orthopedics. 2nd ed. Lea and Febiger, Philadelphia.

Egger E.L. 1993. Fractures of the radius and ulna, p.1736-1757. In: Slatter D. (ed.), Textbook of Small Animal Surgery. 2nd ed. W.B. Saunders, Philadelphia.

Harasen G. 2003. External coaptation of distal radius and ulna fractures. Can Vet. J. 44:1010-1011.

Herron M.R. 1974. Repair of distal radio-ulnar fractures in toys breeds. Can. Pract. 1:12-17.

Johnson K.A. 1981. Retardation of endochondral ossification of distal ulnar growth plate in dogs. Aust. Vet. J. 57:474-476.

Lappin M.R., Aron D.N., Herron H.L. \& Malnati G. 1983. Fractures of the radius and ulna in the dog. J. Am. Anim. Hosp. Assoc. 19:643650.

Piermattei D.L. 1978. Practical orthopedics of long bones and lower forelimb. Proceedings of the American Animal Hospital Association, p.45.

Rovesti G.L., Bosio A., Marcelli-Little D.J. 2007. Management of 49 antebrachial and crural fractures in dogs using circular external fixators. J. Small Anim. Pract. 48(4):194-200.

Sumner-Smith G. 1970. Bone plating for radial fractures in small dogs. Modern Vet. Pract. 3:30-33.

Sumner-Smith G. \& Cawley A.J. 1970. Nonunion of fractures in the dog. J. Small Anim. Pract.11:311-325.

Turner M.T. 1995. Fractures of the bone of the front limb, p.195-219. In: Olmstead M.L. (ed.), Small Animal Orthopedics. Mosby-Year Book, St Louis.

Vaughan L.C. 1984. A clinical study of nonunion fractures in the dog. J. Small. Anim. Pract. 5(8):173-177. 